Methods We collected dietary information for 171 children aged 4-5 years from parents of the INMA study in Valencia, a prospective mother-child cohort study. A 105 food items FFQ was used to assess the child's diet of previous year, at baseline and, on average, 10 months later. As the reference method, we estimated several carotenoids, vitamin $\mathrm{C}$ and $\mathrm{E}$ in the plasma of the children. Pearson (and Spearman) correlations were calculated for reproducibility (FFQ1 vs FFQ1), and validity by comparing nutrient estimates from FFQ to nutrient biomarkers (biochemical calibration).

Results The average of correlation coefficients for reproducibility (Spearman r) between the two FFO was 0.44 (0.44 for energy, 0.41 for protein, 0.39 for carbohydrate, 0.41 fat, 0.55 for $\beta$-carotene, 0.60 for vitamin $\mathrm{C}$ and 0.39 for vitamin $\mathrm{E}$ ). The average of correlation coefficients for validity (Pearson $\mathrm{r}$ ) between the mean of two FFO and nutrients in plasma were 0.06 for $\boldsymbol{\alpha}$-tocopherol, 0.10 for lutein-zeaxanthin, 0.44 for $\beta$-cryptoxanthin $(p<0.001)$, 0.20 for lycopene $(p=0.01), 0.18$ for $\alpha$-carotene $(p=0.021), 0.24$ for $\beta$-carotene $(p=0.002)$ and 0.23 for vitamin $C(p=0.008)$. When children from mothers who reported a change in their children diets were excluded from the analysis correlations were improved.

Conclusions The FFQ showed a relatively good reproducibility and satisfactory agreements with most nutrient biomarkers measured in blood which may support its use as a valid instrument for dietary assessment in preschool children at these early ages.

\section{P1-481 IMPACT OF STUDY DESIGN, PARTICIPANT SELECTION AND ADJUSTMENT METHODS ON ESTIMATED EFFECTS IN NON- RANDOMISED RESEARCH}

doi:10.1136/jech.2011.142976g.70

J Nicholas, ${ }^{*}$ A Grieve, M Gulliford. King's College London, London, UK

Introduction When electronic patient records are used for nonrandomised research, a range of different study designs, eligibility criteria and adjustment methods may be used. This study aimed to compare the apparent bias and precision of effect estimates resulting from different potential design and analysis methods.

Methods Comparisons were based on the association between thiazolidinedione (TZD) therapy and heart failure in 91872 participants with diabetes. Nested within the same dataset, we applied all feasible combinations ( $N=162)$ of: five study designs; five sets of eligibility criteria for sample selection; and eight methods of adjustment. Apparent bias was evaluated by comparison to the RR of $1.72(1.21-2.42)$ from a meta-analysis of RCTs. Precision was evaluated from SEs.

Results The multiple regression adjusted HR from the full sample cohort study was 1.34 (1.15-1.56). Adjusted effect estimates from the case-only study designs had low precision and were higher than the reference value, ranging up to an OR of 8.22 (4.92-13.71) for the case-crossover design. After applying restrictive eligibility criteria (including new-user, and RCT-like criteria) precision was lower and adjusted effect estimates were generally lower than the reference value. Application of new user, propensity score and confounder based exclusions gave the lowest HR of 0.43 (0.10-1.76). Choice of adjustment method had a relatively small impact on the magnitude and precision of the effect estimate.

Conclusion Our results suggest that restricting eligibility criteria, or implementing case-only designs, may not always reduce bias, and may reduce precision, in comparison to a cohort study using the full sample.

\section{P1-482 SLEEP DEPRIVATION AND OVERWEIGHT AMONG SHIFT WORKERS OF A POULTRY SLAUGHTERHOUSE PLANT IN SOUTHERN BRAZIL}

doi:10.1136/jech.2011.142976g.71

M T Olinto, ${ }^{*}$ R Canuto, R Henn, J Macagnan, M Pattussi. University of Vale do Rio dos Sinos, São Leopoldo, RS, Brazil

Nowadays, in many sectors of the economy, society functions $24 \mathrm{~h}$ per day. During last century, there has been a decrease in time that people spend sleeping. Studies have suggested that disruption of circadian rhythm may lead to obesity, we assessed the relation between sleep deprivation and overweight among shift workers. A cross-sectional study was conducted on a sample of 902 workers aged 18 to 50 years who were working on the production line, in a poultry slaughterhouse in Southern Brazil. Overweight (overweight + obese) was defined as body mass index $\geq 25 \mathrm{~kg} / \mathrm{m}^{2}$. Time of sleep was categorised as: $>5 \mathrm{~h}$ continuous/day; $\quad<=5$ h continuous/day with some additional rest (sleep deprivation level I); and $\leq 5 \mathrm{~h}$ /day without any additional rest (sleep deprivation level II). The mean age of the participants was 31 years (SD $=8.7)$, 63\% of the sample were women and $20 \%$ sleep $5 \mathrm{~h}$ or less in continuous/day. Workers with sleep deprivation level II and level I showed higher prevalence of overweight $(66.7 \%$ and $45.7 \%$ vs $37.4 \%$ ) than workers who slept $5 \mathrm{~h}$ or more hours in continuous/day. After adjusting for sociodemographic, parent's overweight, behavioural (meals/day) and shift work (night/day), the prevalence ratios for sleep deprivation level II and level I were, respectively, 1.76 (95\% CI 0.93 to 3.36 ) and 1.24 (95\% CI 0.93 to 1.64) compared with workers sleeping $>5$ h continuous/day. Sleep deprivation may be an independent risk factor for overweight in shift workers.

\section{P1-483 SOCIOECONOMIC STATUS AND PSYCHOLOGICAL DISTRESS: A NATIONWIDE STUDY FROM IRAN}

doi:10.1136/jech.2011.142976g.72

S Omidvari, A Montazeri, * J Sadighi, F Farzadi, F Maftoon, A Aeenparast, K Jahangiri, M Vahdaninia. Iranian Institute for Health Sciences Research, ACECR, Tehran, Iran

Introduction Socioeconomic factors might play important roles in developing psychological distress. As part of a large study on health perception in Iran the relationship between anxiety and socioeconomic factors was investigated.

Methods This was a nationwide cross sectional study. A random sample of individuals aged between 18 and 65 were entered into the study. Respondents were asked to rate their anxiety on a 5-point scale. The record of demographic and socioeconomic data included age, gender, education, marital status, employment, income, and chronic diseases. Logistic regression analysis was used to estimate ORs for contributing factors to self-reported anxiety.

Results In all 27883 individuals took part in the study. The mean age of the respondents was 32.7 (SD=11.8) years. Overall $20.1 \%$ reported that they were very or very much anxious. The results obtained from logistic regression analysis showed that females [OR $=1.52]$, lower income groups [OR for lowest income group $=1.53$ ], having at least one chronic disease $[\mathrm{OR}=1.27]$, and those with lower self-reported health [OR for lowest group $=5.12$ ], were more likely to suffer from poor mental health.

Conclusion The findings indicated that there were inverse relationships between anxiety, income and self-reported health. The contribution of income to psychological distress might be explained in the context of social determinants of health. 